\title{
Patients with precursor disease exhibit similar psychological distress and mental $\mathrm{HRQOL}$ as patients with active myeloma
}

\author{
Imad Maatouk (1,2, Susanne He ${ }^{3}$, Manuela Hummel ${ }^{4}$, Stefan Hemmer ${ }^{5}$, Michaela Hillengass ${ }^{6}$, Hartmut Goldschmidt ${ }^{3,7}$, \\ Mechthild Hartmann', Wolfgang Herzog ${ }^{1}$ and Jens Hillengass ${ }^{3,8}$
}

Multiple myeloma (MM) is largely a disease of the elderly with a median age at first diagnosis of 69 years. MM accounts for $10 \%$ of hematologic malignancies, with an incidence of 5-8/100,000 individuals in the US ${ }^{1}$. Several studies have investigated the impact of $M M$ on health-related quality of life (HRQOL), psychological distress (DT), and mental comorbidity ${ }^{2}$ due to a variety of disease- and treatment-related events leading to impairment in terms of HRQOL and increased $\mathrm{DT}^{3}$.

However, MM is consistently preceded by a monoclonal gammopathy of undetermined significance (MGUS) and smoldering (asymptomatic) MM (SMM), which are premalignant stages of the disease with the presence of monoclonal plasma cells and/or monoclonal protein, but lacking so-called myeloma-defining events ${ }^{4}$.

It is estimated that $\sim 5 \%$ of the elderly population (aged $\geq 70$ years) are diagnosed with MGUS. In individuals aged $\geq 85$ years the prevalence of MGUS in men increases to 9\%. Depending on clinical risk factors, MGUS and SMM require regular consultations, as the annual risk of progression is 1 and 10\% for MGUS and SMM, respectively ${ }^{5}$. Clinicians and patients report that this period of watchful "waiting" characterized by repeated follow-up visits has a high psychosocial impact on patients' quality of life. Pilot data suggest similar or even higher levels of DT in patients with non-malignant hematologic entities ${ }^{6,7}$. Here, we aimed to compare HRQOL, DT, anxiety, and depression between patients with MGUS/SMM and patients with MM pre- and post-treatment.

\footnotetext{
Correspondence: Imad Maatouk (imad.maatouk@med.uni-heidelberg.de) 'Department of General Internal Medicine and Psychosomatics, Medical University Hospital, Heidelberg, Germany

${ }^{2}$ National Center for Tumor Diseases, Heidelberg, Germany

Full list of author information is available at the end of the article.
}

All patients who made the first appointment at our outpatient clinic between November 2014 and September 2017 received a letter prior to their appointment at the hospital. This letter included information on the survey and a psychosocial questionnaire package. Patients with a diagnosis of MM, SMM, or MGUS, and who also had sufficient skills in reading and writing German were eligible for study participation. Written, informed consent for analysis of patient data was obtained from all participants. Approval was obtained from the local ethics committee at the University Hospital of Heidelberg.

HRQOL was measured using the Short Form General Health Survey (SF-12).The items were weighted and totaled to provide both a physical component score (PCS) and a mental component score (MCS) ${ }^{8}$.

DT was measured using the National Comprehensive Cancer Network (NCCN) distress thermometer?.

The nine-item patient health questionnaire (PHQ-9) and the seven-item general anxiety disease-screening instrument (GAD-7) were used to measure depression and anxiety symptoms, respectively ${ }^{10}$.

Participants were classified into three groups based on the guidelines of the International Myeloma Working Group and according to time-point in the course of the disease as follows: new diagnosis of MM (pre-treatment), treated $\mathrm{MM}$, and premalignant disease (MGUS or SMM) $)^{4}$.

Group comparisons (Kruskal-Wallis tests) for the respective outcomes and multivariate regression analyses were performed, with HRQOL, DT, anxiety, and depression scores as outcome measures.

Classification of a disease stage (Group 1: premalignant disease; reference, Group 2: new diagnosis of MM before treatment, and Group 3: treated MM) served as 
independent variable of interest in all multivariate regression analyses. All statistical analyses were performed using $\mathrm{R}$ for Windows (version 3.3.2).

Between November 2014 and September 2017, 552 of 1066 eligible patients (response rate: 51.8\%) were enrolled in the study. The mean age was 62.1 years with a range of $30.7-87.5$ years. The majority of patients were male $(n=$ 324; 58.7\%). Of the 552 patients, 190 (35.8\%) had a new diagnosis of active MM, 193 patients (36.4\%) had already been treated for MM, and 148 patients (27.9\%) had a diagnosis of a precursor (MGUS or SMM) of MM. The mean values of the SF-12 sum scores in the whole cohort were 42.4 for MCS (range 11.5-68.9) and 39.1 for PCS (range 16.4-59.6), respectively. Psychological distress according to the DT level was above the threshold of $\geq 5$ in 338 participants (63.9\%). Moderate to severe anxiety symptoms according to the GAD-7 were prevalent in $12.4 \%$ of participants, while moderate to severe depressive symptoms according to the PHQ-9 were prevalent in 21\% of participants. Characteristics of the entire sample are shown in Table 1.

Group comparison revealed that individuals with a precursor of MM reported higher physical HRQOL scores (PCS: mean 43.6) $(p<0.001)$ than patients with a newly diagnosed symptomatic stage of the disease (PCS: mean 39.1) and already treated patients (PCS: mean 35.7).

No statistically significant difference was found with regard to mental HRQOL in individuals with a precursor of MM (MCS: mean 43.1), newly diagnosed, (MCS: mean 42.9) and treated MM (MCS: mean 41.3) $(p=0.18)$. The outcome parameters of patients stratified by groups $1-3$ with the respective $p$-values of group comparison tests are available as Supplementary Information (SI) on blood cancer journal's website.

The results were confirmed in multivariate analysis. The stage of the disease according to the above-mentioned classification was significantly related to PCS, but was not significantly associated with MCS, respectively. The results of all multivariate linear regression analyses are shown in Table 2.

When comparing psychological distress between patients with a precursor of MM (DT: mean 5.2), newly diagnosed MM (DT: mean 5.7), and treated MM (DT: mean 5.3), no significant differences were found ( $p=$ $0.26)$. In multivariate analysis, compared with the premalignant stages (reference), no statistically significant associations of distress were found between a new diagnosis of MM $(p=0.87)$ or treated MM $(p=0.33)$.

No significant differences were found for anxiety symptom scores between the three groups (MGUS or SMM: mean 4.1/newly diagnosed MM: mean 4.8/treated MM: mean $4.6 ; p=0.21$ ). The results of multivariate analysis confirmed these findings (Table 2). Compared with the premalignant stages (reference), no statistically
Table 1 Patient characteristics

\begin{tabular}{|c|c|c|c|}
\hline Variables & $M(\mathrm{SD})$ & $n$ & $\%$ \\
\hline Age in years & $62.1(10.5)$ & 552 & 100 \\
\hline \multicolumn{4}{|l|}{ Sex } \\
\hline Female & & 228 & 41.3 \\
\hline Male & & 324 & 58.7 \\
\hline \multicolumn{4}{|l|}{ Stage of myeloma } \\
\hline Precusor disease (MGUS. SMM) (Group 1) & & 148 & 27.9 \\
\hline New diagnosis of MM (Group 2) & & 190 & 35.8 \\
\hline Treated MM (Group 3) & & 193 & 36.4 \\
\hline \multicolumn{4}{|l|}{ Comorbidity } \\
\hline Charlson comorbidity index $\leq 2$ & & 404 & 74.1 \\
\hline Charlson comorbidity index $>2$ & & 141 & 25.9 \\
\hline Distress thermometer & $5.4(2.8)$ & 529 & \\
\hline Low $(<5)$ & & 191 & 36.1 \\
\hline High $(\geq 5)$ & & 338 & 63.9 \\
\hline \multicolumn{4}{|l|}{ Health-related quality of life } \\
\hline Physical component score (PCS 12) & $39.1(11.0)$ & 541 & \\
\hline Mental component score (MCS 12) & $42.4(11.6)$ & 541 & \\
\hline Anxiety (GAD-7) score & $4.5(4.4)$ & 532 & \\
\hline \multicolumn{4}{|l|}{ Anxiety level } \\
\hline None (0-4) & & 323 & 60.8 \\
\hline Mild (4-9) & & 142 & 26.7 \\
\hline Medium (10-14) & & 40 & 7.5 \\
\hline Severe (15-21) & & 26 & 4.9 \\
\hline Depression (PHQ-9) score & $5.9(4.6)$ & 542 & \\
\hline \multicolumn{4}{|l|}{ Depression level } \\
\hline None (0-4) & & 247 & 45.6 \\
\hline Mild (4-9) & & 181 & 33.4 \\
\hline Medium (10-14) & & 84 & 15.5 \\
\hline Severe (15-27) & & 30 & 5.5 \\
\hline
\end{tabular}

MM multiple myeloma, MGUS monoclonal gammopathy of undetermined significance, SMM smoldering myeloma, PCS physical component score, MCS mental component score, GAD-7 generalized anxiety disorder scale-7, PHQ-9 patient health questionnaire-9

significant associations of anxiety symptom scores were found between newly diagnosed MM $(p=0.6)$ or treated MM $(p=0.91)$. For depression symptom scores, significant differences were found between the groups (MGUS or SMM: mean 4.9/newly diagnosed MM: mean 5.6/treated MM: mean 6.9; $p<0.001$, respectively). In multivariate regression analysis, there was no association between newly diagnosed MM and depression score $(p=$ 0.98 ), although a significant association was found 


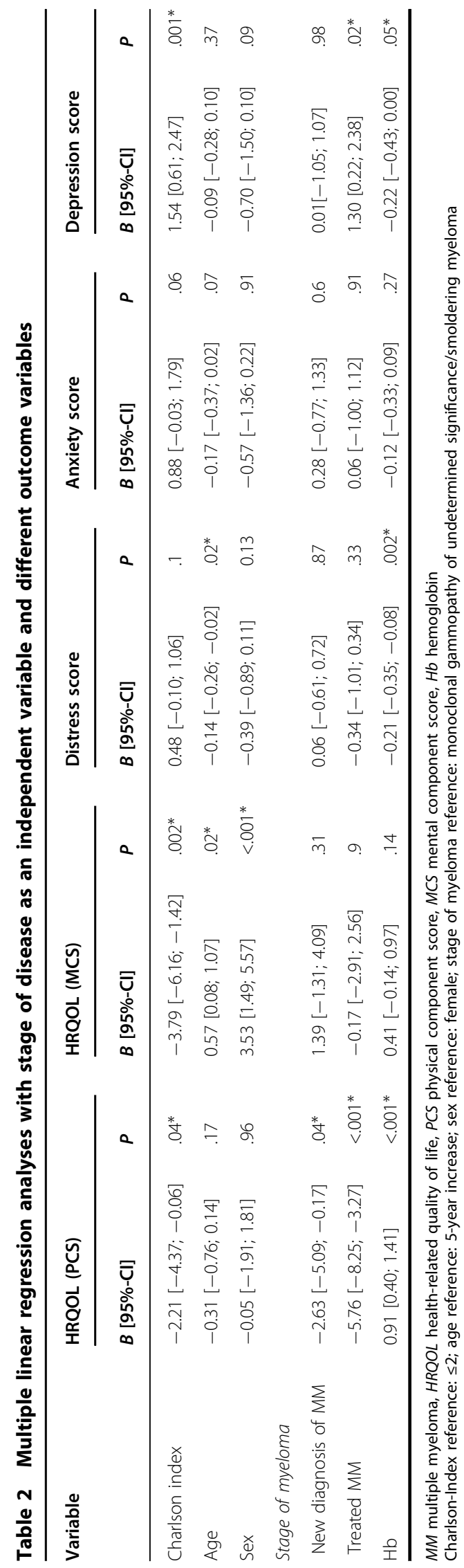

between treated MM $(B=1.3 ; p=0.04)$ when compared with the precursor stages (MGUS/SMM; reference).

To the best of our knowledge, this is the first investigation of HRQOL and various aspects of mental health considering precursor states of $\mathrm{MM}$ in a large clinical sample of over 500 patients. In a recently published article with a smaller sample of 292 participants we investigated the relation between resilience, HRQOL and depression in $\mathrm{MM}$ and its premalignant stages. Patients with a high level of resilience had a better HRQOL. In this study DT and anxiety levels were not considered ${ }^{11}$. Patients' experience in terms of quality of life need to be considered in a group of individuals who have a life expectancy that is not significantly limited by the respective diagnosis, as in the case of MGUS. In an ongoing longitudinal study, launched in November 2016 in Iceland, aiming to include $\sim 120,000$ adults for a screening to identify precursors of $\mathrm{MM}$, one aim is to determine the optimal diagnostic procedures for individuals with MGUS. Longitudinal investigations of HRQOL are to be included as part of this study. However, the results will not be available in the near future ${ }^{12}$.

In our study, patients with MM reported lower levels of physical HRQOL compared with patients with a precursor disease. Lowered levels of PCS in patients with MM compared with precursors in our sample can be explained by the typical symptoms of active MM, including fatigue and pain, or by treatment-related side effects $^{13}$.

In our study, no significant differences in mental HRQOL, distress, or severity of anxiety symptoms were found between the precursors and MM groups in univariate or multivariate analyses. This is in line with the reports of clinicians that the psychological burden of cancer anticipation and screening procedures in MGUS leads to similar psychological burdens for patients with MGUS, SMM, and MM alike 6 .

In our sample, $\sim 63 \%$ of all participants reported levels of DT above the cutoff-score of $\geq 5$ with no significant differences between subgroups, suggesting a need for psychosocial support in the majority of patients.

The prevalence of clinically relevant anxiety symptoms in MM was comparable to other studies in the field ${ }^{14}$. The fact that there was no significant differences between precursors and active MM suggests the need to screen for psychological burden in this vulnerable group.

Levels of depression symptoms were significantly higher in the group with treated myeloma than in the precursor group. This may be due to treatment-related adverse events and disease-related symptoms.

The major strength of the present study is the large clinical sample. HRQOL, DT, anxiety, and depression were measured with validated questionnaires. The impact of our findings is magnified by the fact that the clinical 
prevalence (diagnosed cases) of MGUS is huge-estimated to be $>500,000$ in the US alone ${ }^{15}$.

The main limitation is related to its cross-sectional design, which does not allow for either temporal or causal inferences.

Our findings support the perception of many clinicians who report patients with MGUS and SMM suffering significantly from the knowledge of their risk of developing MM. Therefore, even in patients with a precursor disease, it may be important to routinely measure DT in order to provide needs-based support.

\section{Acknowledgements}

We acknowledge financial support by Deutsche Forschungsgemeinschaft within the funding programme Open Access Publishing, by the BadenWürttemberg Ministry of Science, Research and the Arts and by Ruprecht-KarlsUniversität Heidelberg.

\section{Author details}

'Department of General Internal Medicine and Psychosomatics, Medical University Hospital, Heidelberg, Germany. ${ }^{2}$ National Center for Tumor Diseases, Heidelberg, Germany. ${ }^{3}$ Department of Hematology, Oncology, and Rheumatology, Medical University Hospital, Heidelberg, Germany. ${ }^{4}$ Department of Biostatistics, German Cancer Research Center, Heidelberg, Germany. ${ }^{5}$ Department of Orthopedics and Traumatology, University Hospital, Heidelberg, Germany. ${ }^{6}$ Roswell Park Comprehensive Cancer Center, Clinical Research Service, Buffalo, NY, USA. ${ }^{7}$ National Center for Tumor Diseases, Section of Multiple Myeloma, Heidelberg, Germany. ${ }^{8}$ Department of Medicine, Roswell Park Comprehensive Cancer Center, Buffalo, NY, USA

\section{Conflict of interest}

The authors declare that they have no conflict of interest.

\section{Publisher's note}

Springer Nature remains neutral with regard to jurisdictional claims in published maps and institutional affiliations.

Supplementary Information accompanies this paper at (https://doi.org/ 10.1038/s41408-019-0172-1).

Received: 7 November 2018 Revised: 27 December 2018 Accepted: 7 January 2019
Published online: 21 January 2019

\section{References}

1. Myeloma - Cancer Stat Facts. SEER Cancer Stat Facts: Myeloma. National Cancer Institute. https://seer.cancer.gov/statfacts/html/mulmy.html. Accessed 2 July 2018

2. Lamers, J. et al. Psychosocial support in patients with multiple myeloma at time of diagnosis: who wants what? Psychooncology 22, 2313-2320 (2013).

3. Kvam, A. K. \& Waage, A. Health-related quality of life in patients with multiple myeloma - does it matter? Haematologica 100, 704-705 (2015).

4. Rajkumar, S. V. et al. International Myeloma Working Group updated criteria for the diagnosis of multiple myeloma. Lancet Oncol. 15, e538-548 (2014).

5. Kyle, R. A. et al. Clinical course and prognosis of smoldering (asymptomatic) multiple myeloma. N. Engl. J. Med. 356, 2582-2590 (2007).

6. Go, R. S. \& Rajkumar, S. V. How I manage monoclonal gammopathy of undetermined significance. Blood 131, 163-173 (2018).

7. Hildebrandt, M. A. T. et al. Quality of life and cancer worry in a follow-up cohort of patients with asymptomatic monoclonal gammopathies. J. Clin. Oncol. 36, 8049 (2018)

8. Ware, J., Kosinski, M. \& Keller, S. D. A 12-Item Short-Form Health Survey: construction of scales and preliminary tests of reliability and validity. Med. Care 34, 220-233 (1996).

9. Carlson, L. E., Waller, A. \& Mitchell, A. J. Screening for distress and unmet needs in patients with cancer: review and recommendations. J. Clin. Oncol. 30, 1160-1177 (2012)

10. Kroenke, K., Spitzer, R. L., Williams, J. B. W. \& Löwe, B. The patient health questionnaire somatic, anxiety, and depressive symptom scales: a systematic review. Gen. Hosp. Psychiatry 32, 345-359 (2010).

11. Maatouk, I. et al. Association of resilience with health-related quality of life and depression in multiple myeloma and its precursors: results of a German crosssectional study. BMJ Open 8, e021376 (2018).

12. pjóðarátak gegn mergæxlum. Leggðu okkur lið. http://www.blodskimun.is. Accessed 5 September 2018.

13. Baz, R. et al. Development of a conceptual model to illustrate the impact of multiple myeloma and its treatment on health-related quality of life. Support. Care Cancer 23, 2789-2797 (2015).

14. Sherman, A. C. et al. Use of a supportive care team for screening and preemptive intervention among multiple myeloma patients receiving stem cell transplantation. Support. Care Cancer 11, 568-574 (2003).

15. Go, R. S., Swanson, K. M., Sangaralingham, L. R., Habermann, E. B. \& Shah, N. D. Clinical prevalence (diagnosed cases) of monoclonal gammopathy of undetermined significance in the US: estimating the burden on health care. Leukemia 30, 1443-1446 (2016). 